\title{
ESTUDO EPIDEMIOLÓGICO E AVALIAÇÃO DA QUALIDADE DE VIDA DOS PORTADORES DE CÂNCER DE LARINGE ATENDIDOS NA UNIDADE DE ALTA COMPLEXIDADE EM ONCOLOGIA (UNACON) DE FEIRA DE SANTANA-BA
}

\author{
$\underline{\text { Jaíne Rocha e Silva }}{ }^{1}$; Márcio Campos Oliveira ${ }^{2}$; Tércio Guimarães Reis ${ }^{3}$
}

1. Bolsista Probic/Uefs, Graduanda em Odontologia, Universidade Estadual de Feira de Santana, email:

jainerocha@outlook.com

2. Orientador, Departamento de Saúde, Universidade Estadual de Feira de Santana, email:

$$
\text { marciopatologiaoral@gmail.com }
$$

3. Participante da pesquisa, Cirurgião de cabeça e pescoço (UNACON), Universidade

Estadual de Feira de Santana, email:

terciohns@gmail.com

\section{PALAVRAS-CHAVE: Qualidade de vida; câncer de cabeça e pescoço; câncer de laringe.}

\section{INTRODUÇÃO}

De acordo com as estimativas globais, o câncer é a segunda causa de morte no mundo, tanto em países em desenvolvimento quanto nos desenvolvidos. Nos Estados Unidos, em 2013, foram registrados 12.260 casos de câncer de laringe, um dos sítios primários em câncer de cabeça e pescoço (CCP) mais frequentemente acometidos no país (American Cancer Society, 2013).

No Brasil, segundo as estimativas do INCA (Instituto Nacional do Câncer) para o ano de 2014, são esperados 576.000 novos casos de câncer. Seguindo-se o panorama mundial, na região de CP destaca-se o de laringe, com 6.870 casos novos, ficando em sétimo lugar geral entre os homens e apenas 770 casos em mulheres, ocupando o $17^{\circ}$ lugar (Brasil, 2013).

Durante os últimos anos, a avaliação da qualidade de vida foi reconhecida como um importante medidor de sobrevida em medicina, sobretudo em oncologia. Tais avaliações tem sido feitas com certa regularidade nos tumores malignos da região de cabeça e pescoço, uma vez que nessa localização a ocorrência de neoplasias malignas pode levar ao comprometimento de funções vitais (Abendstein et al, 2005; Vartanian et al, 2006). Nesse sentido, em 1993, o World Health Organization - Quality of Life Group (WHOQOL), definiu qualidade de vida como: percepção individual da posição do indivíduo na vida, no contexto de sua cultura e sistema de valores nos quais ele está inserido e em relação aos seus objetivos, expectativas, padrões e preocupações. É um conceito de alcance abrangente afetado de forma complexa pela saúde física, estado psicológico, nível de independência, relações sociais e relações com as características do meio ambiente do indivíduo (Moreno \& Lopes, 2002). Incontestavelmente, a presença de qualquer neoplasia altera todos os aspectos da vida de uma pessoa e pode levar a intensas mudanças dos seus hábitos.

\section{METODOLOGIA}

Trata-se de um estudo transversal de caráter descritivo, que foi realizado na Unidade de Alta Complexidade em Oncologia (UNACON), de Feira de Santana, BA. A população 
estudada foi constituída de pacientes portadores de câncer de laringe em tratamento nas mais variadas modalidades, no período compreendido entre setembro de 2016 e agosto de 2017.Foram incluídos na pesquisa todos os pacientes portadores de câncer de laringe atendidos na UNACON de Feira de Santana - BA, que tinham idade igual ou superior a 18 anos e que aceitaram participar do estudo.O procedimento de coleta foi realizado apenas por uma pesquisadora previamente treinada e calibrada para tal procedimento e processamento dos dados obtidos. O instrumento de coleta dos dados consistiram do prontuário dos pacientes para a obtenção dos dados epidemiológicos como idade, sexo, renda familiar, nível de escolaridade, etc. e de um questionário de Avaliação da Qualidade de Vida da Universidade de Washington (UW-QOL) em pacientes portadores de neoplasias malignas de cabeça e pescoço, devidamente validado para o idioma português (VARTANIAN et al, 2007). Neste questionário foram avaliadas as condições de saúde geral do paciente, atividade, recreação, dor, humor e ansiedade relativas à doença. Os dados foram avaliados descritivamente por meio da apresentação das frequências absolutas e percentuais relativas. O programa utilizado para a compilação dos dados foi o Statistical Package for Social Science - SSPS versão 10.0. Posteriormente, as informações foram sistematizadas em tabelas e gráficos com o auxílio do Programa Excel da MICROSOFT CORPORATION (2007), obedecendo a sequência e distribuição das variáveis do estudo, para apresentação dos resultados obtidos.

\section{RESULTADOS}

A prevalência do tumor de laringe foi de $25,8 \%$, correspondendo a 8 casos em uma amostra de 31 pacientes com câncer de cabeça e pescoço. Houve uma prevalência maior no sexo masculino, equivalendo a $75 \%$ dos casos. A média de idade foi de 60,75 anos, sendo a menor 33 e a maior 78. Dentre os participantes, metade eram casados e a outra metade solteiros.Quanto às demais características sócio-demográficas, $25 \%$ dos participantes eram analfabetos, $37,5 \%$ sabiam apenas ler e escrever, $25 \%$ concluíram o ensino fundamental e $12,5 \%$ cursaram o nível superior, enquanto que $77,5 \%$ dos pacientes não terminaram o ensino médio. Quanto à ocupação, $62,5 \%$ já se encontram aposentados e $50 \%$ não trabalham devido ao estado de saúde. A renda mensal das famílias é de 1 salário mínimo para $50 \%$ dos casos. A cidade de procedência foi igual para Feira de Santana e localidades vizinhas, sendo a área urbana mais prevalente (75\%).Em relação aos hábitos de vida, $75 \%$ e $87,5 \%$ dos pacientes eram fumantes e etilistas respectivamente. Em relação ao estadiamento da doença, houve prevalência de tumores em estadio T4 (37,5\%) N2c (25\%) e M0 (75\%).A qualidade de vida global dos pacientes foi analisada somando-se a pontuação de cada domínio do questionário UW-QOL (escore total), o que, portanto, pode variar de 0 a 1200, já que são 12 domínios, cada um podendo ser pontuado de 0 a 100. A média encontrada foi de 821 pontos. O paciente com a melhor qualidade de vida pontuou 1167, enquanto que o paciente com pior qualidade de vida pontuou 424.

Dentre os doze domínios do questionário UW-QOL, as variáveis que apresentaram o menor escore foram: atividade, paladar, recreação, humor, ansiedade, fala, dor e mastigação. Saliva, aparência, deglutição e ombro apresentaram as maiores pontuações respectivamente.

Quando questionados sobre quais problemas estavam sendo mais importantes nos últimos 7 dias, a dor e o paladar foram os mais relatados pelos pacientes. Comparada com o mês anterior ao de desenvolvimento do câncer, 5 pacientes classificaram sua qualidade de vida relacionada a saúde em mais ou menos a mesma, $1 \mathrm{em}$ um pouco melhor, $1 \mathrm{em}$ ruim e 1 em muito pior. Quanto à qualidade de vida geral, 4 consideraram como média, 2 como boa e 2 como muito ruim. 


\section{DISCUSSÃO}

Epidemiologicamente os dados obtidos neste estudo estão de acordo com a maioria dos relatos encontrados na literatura, onde a prevalência do câncer de laringe é maior em indivíduos do sexo masculino, com uma faixa etária acima de 55 anos (Moreno \& Lopes, 2002). Entretanto, é importante ressaltar que se observou uma precocidade de ocorrência do tumor em alguns indivíduos (33 anos), o que corresponde a 12,5\% da amostra estudada.

A grande maioria dos participantes fez uso durante muito tempo (mais de uma década) do tabaco e/ou do álcool, o que corrobora com vários estudos, onde estes são tidos como os principais fatores de risco para o desenvolvimento do câncer de cabeça e pescoço, sendo responsáveis por quase $90 \%$ dos casos (Inca, 2009).

Outro fato observado foi a baixa escolaridade dos pacientes, pois apenas 1 paciente havia completado o ensino médio, enquanto dois eram analfabetos. Segundo a literatura, isso pode colaborar para o atraso no diagnóstico e aumento da dificuldade de compreensão e sucesso do tratamento (Maybury, 2012).

O prognóstico dos pacientes com câncer de laringe está intimamente relacionado ao estadiamento do tumor. Neste estudo, a esmagadora maioria dos participantes estavam em estádio avançado (T3 e T4), em comparação aos estádios precoces (T1 e T2), onde se observou um caso apenas (Rapoport, 2001; Herchenhorn, 2004).

$\mathrm{Na}$ avaliação do UW-QOL, pôde-se perceber que os domínios mais afetados pelo câncer de laringe e, portanto, os que tiveram menor pontuação foram: em primeiro lugar a atividade com (50 pontos), o paladar foi a segunda variável mais afetada ( 54,25 pontos). A explicação pode estar contida no fato de que o tabaco aumenta a descamação da gengiva, destruição do tecido e pode ter como consequência sérios problemas periodontais, ocorrendo alteração no paladar(Inca, 2001). O humor e a recreação foram os terceiros domínios mais atingidos (59,38 pontos), provavelmente pelo momento em que o questionário foi aplicado, o qual corresponde ao mesmo do diagnóstico. Essa explicação também se considera para a ansiedade (Sommerfeld et al, 2012), que obteve pontuação de (59,5). Mastigação e dor $(68,75$ pontos) foram o quarto domínio com pior pontuação. A dificuldade na mastigação pode estar relacionada com a localização do tumor. Os que obtiveram maior pontuação e, consequentemente, foram os menos afetados são os seguintes: a saliva (87,5 pontos), aparência ( 84,38 pontos), deglutição ( 83,38 pontos) e ombro ( 79,13 pontos).

\section{CONCLUSÃO}

A qualidade de vida é um aspecto subjetivo que envolve a consideração de aspectos sociais, ambientais e psicológicos. Neste estudo, a qualidade de vida dos portadores de câncer de laringe se mostrou afetada tanto antes como após o diagnóstico, e a realização de estudos nessa área contribui para o desenvolvimento de estratégias que podem melhorar $o$ atendimento desses pacientes na esfera multidisciplinar, propiciando assim, uma melhoria na saúde e qualidade de vida dessas pessoas

\section{REFERÊNCIAS}

AMERICAN CANCER SOCIETY. Cancer Facts \& Figures 2013. Atlanta: American Cancer Society; 2013

BRASIL. Ministério da Saúde. Instituto Nacional de Câncer. Estimativa 2014: incidência de câncer no Brasil. Rio de Janeiro: Inca; 2013. 
ABENDSTEIN, H.; NORDGREN, M.; BOYSEN, M.; JANNERT, M.; SILANDER, E; AHLNER-ELMQVIST, et al. Quality of Life and Head and Neck Cancer: A 5 Year Prospective Study. Laryngoscope, v. 115, p. 2183-2192, 2005.

VARTANIAN, J.G.; CARVALHO, A.L.; YUEH, B.; FURIA, C.L.B.; TOYOTA, J.; MCDOWELL, J.A. et al. Brazilian- portuguese validation of the university of washington quality of life questionnaire for patients with head and neck cancer. Head \& Neck, v.28, p. 1115-1121, 2006

MORENO, A.B.; LOPES, C.S. Avaliação da qualidade de vida em pacientes laringectomizados: uma revisão sistemática. Cad. Saúde Pública, v.18, n.1, p. 81-92. 2002.

BRASIL. Ministério da Saúde. Instituto Nacional de Câncer - INCA.Estimativa 2010: incidência de câncer no Brasil. Rio de Janeiro: INCA 2009. 98 p.

MAYBURY, C; HOROWITZ, A.M; GOODMAN, H.S. Outcomes of oral cancer early detection and prevention statewide model in Maryland. J Public Health Dent. 2012; 72 Suppl 1:S34-8. doi: 10.1111/j.1752- 7325.2012.00320.x.

RAPOPORT, A, KOWALSKI, L.P; HERTER, N.T; BRANDÃO, L.G; WALDER, F. Projeto Diretrizes - Câncer de Boca. Associação Médica Brasileira e Conselho Federal de Medicina, 2001.

HERCHENHORN, D; Dias F.L. Avanços no tratamento quimioterápico e radioterápico do câncer de cabeça e pescoço. Rev. Hosp. Clin. 2004,59 (1), pp. 39-46.

CONDUTAS DO INCA/MS. Carcinoma epidermóide da Cabeça e Pescoço.

Revista Brasileira de Cancerologia,2001 47(4): 361-37.

SOMMERFELD et al. Rev. Bras. Cir. Cabeça Pescoço, v.41, $\mathrm{n}^{\circ}$ 4, p. 172-177, outubro / novembro / dezembro 2012 